\title{
Los procesos participativos locales en la producción de la ciudad. Experiencia del Taller Internacional de Diseño Urbano en Ayacucho, Perú ${ }^{1}$
}

\section{Local participatory processes at the service of urban design. A case study of the International Urban Design Workshop in Ayacucho, Peru}

\author{
María Antonia Ñope Cueva \\ https://orcid.org/0000-0002-4139-7994 \\ maria.nope@unmsm.edu.pe \\ Universidad Nacional Mayor de San Marcos
}

\author{
Liliana Mercedes Murga Riva \\ https://orcid.org/0000-0003-2666-8909 \\ liliana.murga@unmsm.edu.pe \\ Universidad Nacional Mayor de San Marcos
}

\begin{abstract}
RESUMEN
A partir de la experiencia de movilización de actores del Taller Internacional de Diseño Urbano (TIDU) del proyecto Urban-Andes, en la ciudad de Ayacucho (2019), se busca reflexionar sobre los procesos participativos y la aplicación de metodologías participativas en los procesos locales. El marco de análisis se construye sobre los postulados de la Investigación Acción Participativa, así como la interculturalidad y lo glocal. El TIDU es un espacio para dialogar, deliberar y trabajar sobre propuestas de diseño urbano que respondan a una problemática local. Sin embargo, la experiencia plantea el desafío de la participación social en un contexto de debilidad institucional y desigualdades sociales persistentes.
\end{abstract}

Palabras clave: Participación Local; Investigación Acción; diseño urbano; Ayacucho.

\section{ABSTRACT}

Drawing from the mobilization of stakeholders around the International Urban Design Workshop (IUDW) conducted in Ayacucho in 2019, this paper aims to reflect on participatory processes and the application of participatory methodologies in local processes. The analysis builds upon the Participatory Research-Action framework and the concepts of interculturalism and "glocalization". The IUDW promotes dialogue, deliberation, and exchange of knowledge among stakeholders as a strategy to generate urban design intervention proposals that address local issues. Despite the methodology's promises, this case study highlights the challenge of social participation in a context of weak institutions and persistent social inequalities.

Keywords: Local participation; Action-Research; urban design; Ayacucho.

\footnotetext{
1 Queremos agradecer a Pedro Jacinto, profesor de la UNMSM, y Simon Laflamme, colaborador del TIDU 2019, por los aportes y comentarios brindados durante la elaboración del presente artículo.
}

(c) Los autores. Este artículo es publicado por ISHRA, Revista del Instituto Seminario de Historia Rural Andina de la Facultad de Ciencias Sociales de la Universidad Nacional Mayor de San Marcos. Este es un artículo de acceso abierto, distribuido bajo los términos de la licencia Creative Commons Atribución 4.0 Internacional (CC BY 4.0) [https://creativecommons.org/licenses/by/4.0/deed.es] que permite el uso, distribución y reproducción en cualquier medio, siempre que la obra original sea debidamente citada de su fuente original. 


\section{Introducción}

Hoy más que nunca, la gestión de las ciudades es clave para hacer frente a los retos que conlleva el crecimiento de la población urbana. En Perú, las ciudades han crecido de forma desordenada, en muchos casos mediante la ocupación informal de tierras. Esto ha venido acompañado de desigualdades crecientes dentro de las ciudades, en el acceso a servicios básicos, a espacios públicos y a oportunidades económicas.

Ayacucho es una ciudad andina, mayoritariamente quechua hablante y poseedora de una cultura reconocida a nivel nacional. De acuerdo con el INEI (2017), entre 1940 y 2017, su población se multiplicó por 12 . Con una tasa anual de $2,5 \%$, su crecimiento demográfico es superior al promedio nacional, generando varios de los retos mencionados anteriormente. En particular, la expansión física de la ciudad está entrando en conflicto con barreras naturales, afectando su sostenibilidad medioambiental. Este crecimiento se enmarca en una pobreza económica $^{2}$ y una vulnerabilidad social agudizada durante la violencia política social en la década de los ochenta.

En 2019, se realizó en Ayacucho el Taller Internacional de Diseño Urbano Paisajístico $(\mathrm{TIDU})^{3}$. Durante 10 días, se reunieron equipos multidisciplinarios locales e internacionales a fin de elaborar propuestas para solucionar las principales problemáticas de sostenibilidad urbana. En el TIDU se involucraron gobiernos locales, organizaciones de la sociedad civil y la población local. Como resultado, se generaron ideas de intervención de diseño urbano-paisajístico, incorporando elementos como la gestión del agua, la cultura local y la gestión comunitaria.

Este artículo tiene como objetivo principal dar a conocer la importancia de los procesos participativos en un contexto intercultural y glocal, tomando como referencia el proceso participativo que impulsó el TIDU, entre mayo y octubre de 2019. Por glocal, queremos dar cuenta de la relevancia de procesos globales, como son el cambio climático y la urbanización, en la generación de soluciones adaptadas al contexto local. Por intercultural, enfatizamos la interacción entre grupos sociales de diferentes culturas.

Para centrar la discusión, se plantean dos preguntas: ¿De qué manera se aborda la aplicación de metodologías participativas en el ámbito local? y ¿qué retos traen consigo los procesos participativos desarrollados en un contexto intercultural, como el de Ayacucho? Nuestra hipótesis es que los procesos participativos aplicados en contextos interculturales y glocales conllevan retos en el ejercicio de ciudadanía y sostenibilidad. A su vez, la participación en sí contribuye al buen funcionamiento de las instituciones y consolida el tejido organizativo comunitario, lidiando con barreras geográficas y sociales que influyen en la participación.

Para abordar esta investigación emplearemos una metodología cualitativa, especialmente la descripción etnográfica del proceso de participación social basada en la experiencia de una de las autoras como co-organizadora del TIDU. Además, se emplea como marco de análisis los conceptos de metodologías participativas y los procesos participativos, enfatizando en la Investigación Acción Participativa (IAP).

2 En el 2016, en Ayacucho, la incidencia de pobreza fluctuaba entre 32,4\% y 36,1\%, y de pobreza extrema entre $7.7 \%$ y $9.8 \%$. (Instituto Nacional de Estadística e Informática, 2018)

3 El TIDU fue una iniciativa impulsada por la ONG Centro de Competencias del Agua (CCA), la Universidad KU Leuven de Bélgica y la Universidad Nacional San Cristóbal de Huamanga (UNSCH). Financiada por Vlir-Uos, a través del proyecto South Initiative Urban-Andes: Landscape Urbanism Strategies for Resilient Andean Cities in the face of Climate Change; USAID, a través del proyecto PEER Agua-Andes: Ecological Infrastructure Strategies for enhancing Water Sustainability in the Semiarid Andes y Lloyd's Register Foundation, a través del International Water Security Network-IWSN, liderada por la University of the West of England. 
Finalmente, se busca reflexionar sobre la aplicación práctica de las metodologías participativas, así como los retos/desafíos pendientes para un involucramiento real y efectivo de la población.

\section{Marco conceptual y referencial}

Desde las ciencias sociales, la participación ha sido objeto de análisis desde mediados del siglo $\mathrm{XX}$. Aunque existen diferentes maneras de abordar la participación y el involucramiento de la población en los procesos locales, regionales, nacionales e internacionales, aquí se busca resaltar la participación desde la Investigación Acción Participativa (IAP). Este enfoque permite comprender, de una manera interactiva, la participación como un proceso continuo de acciónreflexión, o de compromiso-acción que cobra gran importancia cuando se busca la transformación social de los territorios.

Para sentar las bases conceptuales se esbozan algunas definiciones de los procesos participativos, la metodología participativa y las variables que acompañan su análisis como la interculturalidad y la glocalización.

\section{Las metodologías participativas}

Las metodologías participativas de investigación social surgen a consecuencia de las transformaciones de las ciencias sociales a nivel teórico y epistemológico en el siglo XX, con la conformación del paradigma crítico superando el positivismo y el paradigma interpretativo, por los aportes de la Escuela Crítica de Frankfurt. En la década del setenta, se piensa en una ciencia social que ofrezca un cambio social desde el interior de las comunidades con la finalidad, en el fondo, de la transformación de las estructuras (de las relaciones sociales) dando alternativas a problemas generados a partir de ellas.

En cuanto al paradigma socio-crítico, Popkewitz (1998) destaca el conocimiento de la realidad como praxis, uniendo la teoría y la práctica integrando el conocimiento y la acción, orientando el conocimiento hacia la liberación y emancipación del ser humano, y proponiendo la integración de todos los participantes. Por otro lado, desde la teoría crítica, Habermas (1994) destaca el interés por la racionalidad de la especie humana. En ese sentido, para Alvarado \& García (2008), el paradigma pretende que las ciencias sociales apunten a ofrecer al individuo su propia concientización respecto a sus objetivos y propósitos verdaderos que han sido reprimidos.

Mientras tanto, en América Latina, destacaron las figuras de Paulo Freire (1970) y Orlando Fals-Borda (1970), quienes desarrollaron los conceptos de Investigación Acción Participativa (IAP) en contraste con lo que se venía trabajando en EE.UU., llamado investigación acción (IA). A fines de la década del sesenta a raíz del incremento de la población popular urbana y la masificación de la educación pública, se consolidó una intelectualidad de izquierda, en donde principalmente se critica el sentido funcionalista de las ciencias sociales y se habla de otro tipo de investigación social. En ese escenario surge la IAP, planteando una ciencia social comprometida con la transformación con una relación más respetuosa con la gente y participación en las dinámicas sociales. Esto es lo que en el legado de Fals Borda se conoce como compromiso-acción:

un compromiso con las luchas populares y la transformación social. Compromiso entendido como "la acción o la actitud del intelectual que, al tomar conciencia de su pertenencia a la sociedad y al mundo de su tiempo, renuncia a una posición de simple espectador y coloca su pensamiento o su arte al servicio de una causa". (Como se citó en Lois, 2017, p. 89) 
En síntesis, la IAP se basa en principios como la implicación académica, la no objetificación, la escucha y la creación de una agenda común, el diálogo de saberes y la construcción de poder social vinculando lo académico y lo político (Merçon, 2018). Así mismo, toma elementos del enfoque decolonial por su crítica a las prácticas colonialistas que se manifiestan en la vida individual y colectiva; en donde destaca Anibal Quijano (1999) por su vasta reflexión sobre la colonialidad del poder, quien cuestiona las formas de control y explotación entre grupos dominantes y dominados.

\section{Los procesos participativos}

Tradicionalmente se comprende a la participación como el ejercicio de ciudadanía, en la emisión del sufragio. Es el proceso en que distintos actores de la sociedad median con el Estado para intervenir en asuntos colectivos. Esta ha sido planteada como un derecho básico de todo ser humano (Canto Chac, 2008). Desde los últimos años, se reconoce su legitimidad gerencial y macroeconómica, debido a que produce resultados que van más allá de un discurso, sino que es una oportunidad para incidir en las políticas públicas. Además, desde hace algunas décadas organismos de cooperación internacional como el Banco Interamericano de Desarrollo y Banco Mundial, la consideran una estrategia para el desarrollo humano de la población de América Latina (Kliksberg,1998). Para fines de este artículo se comprende a la participación como un proceso que comprende acciones encadenadas o secuenciales que se ejecutan durante un tiempo determinado por actores organizacionales, institucionales y sociales en coordinación con un gobierno municipal o local.

Los procesos participativos emplean elementos de la metodología participativa para promover el involucramiento activo de todos los miembros en la toma de decisiones, así como en la búsqueda de soluciones a problemas específicos. Con esta premisa se desarrollan talleres, dinámicas de grupo, reuniones, exposiciones interactivas, foros o grupos de trabajo; los cuales están relacionados con la participación de diferentes agentes y/o actores sociales, quienes aportan desde diferentes perspectivas o visiones en relación a un tema o un problema sobre el que se quiere tomar decisiones.

Durante las últimas décadas los gobiernos locales han generado procesos participativos en la ejecución de presupuestos participativos, planes estratégicos municipales, planes sectoriales, planes urbanísticos, etc; a través de mecanismos como talleres (mecanismo de participación donde se encuentran uno o diversos grupos de personas para discutir y evaluar posibles escenarios, propuestas de actuación, etc.), audiencias, referéndum, consulta ciudadana (mediante sistemas de seguimiento y análisis basados en investigaciones cualitativas y cuantitativas), participación vía medios de comunicación masivos, asambleas ciudadanas, entre otros. Es frecuente apreciar en el escenario nacional que la debilidad institucional de los gobiernos locales ha influenciado para que la participación ciudadana no cuente con políticas locales adecuadas que aseguren su eficacia, transparencia y sostenibilidad.

Según la CEPAL (1998), los países de América Latina tienen la necesidad de desarrollar la participación en los asuntos públicos y sociales, de manera que los individuos se transformen en sujetos activos y sean capaces de influir por sí mismos en sus destinos. Sujetos capaces de cuestionar el impacto de su propia participación en la mejora de los modelos institucionales de prestación de servicios públicos, el acceso y la calidad de estos. En esa línea es importante resaltar que la participación es un derecho que demanda generar condiciones de igualdad y respeto por la diversidad étnica y cultural de las sociedades, en especial de los pueblos indígenas de acuerdo al Convenio 169 sobre Pueblos Indígenas y Tribales de la Organización Internacional del Trabajo. 
De acuerdo con la Carta Iberoamericana de Participación Ciudadana en la Gestión Pública (CLAD, 2009), la participación es un proceso de doble vía que requiere, por un lado, entes y organismos públicos receptivos a las opiniones y propuestas de la sociedad; y que, por otro, ciudadanos/as, las comunidades, pueblos indígenas y colectivos sociales conozcan, dialoguen y deliberen las competencias de las instituciones estatales. Esto último hace un llamado a la planificación multiescalar de participación; es decir, entre los diferentes niveles de gobierno. (Sandoval, Sanhueza \& Williner, 2015)

El proceso participativo en su diseño comprende también técnicas propias de la metodología cualitativa y cuantitativa, más allá de las técnicas de la metodología participativa. Con el fin de conocer y comprender al sujeto involucrado en el proceso y a su entorno. En ese sentido, los procesos participativos buscan la transformación o superación de una problemática mediante la interacción entre aquellos que impulsan el proceso y los actores involucrados, y potenciar así su participación para su propio desarrollo. Por ello, el proceso participativo tiene una motivación: el ¿para qué?, que enfatiza el sentir de la lucha social en contra el determinismo social que limita la capacidad de desarrollo y emancipación de las personas y las comunidades.

Entonces, la IAP promueve el empoderamiento de la población mediante su participación, y resalta su rol activo en la transformación social de sus territorios. La IAP como método, plantea un conjunto de etapas que se desarrollan de manera cíclica, pues es un proceso de continua reflexión-acción que empieza por donde termina.

Basados en Suarez \& Meneses (2019), las etapas de un proceso participativo son: la de Diseño del proceso, el Diagnóstico contextualizado, las Devoluciones creativas o talleres, el Planeamiento del rumbo y elaboración del Plan de Acción, la de Ejecución y monitoreo del proyecto y, finalmente, la de Evaluación. En la primera etapa el equipo técnico a cargo del proceso y/o proyecto, identificará, junto con los actores sociales, las características del proceso que implementarán. Para lo cual, en la segunda etapa, pueden utilizar técnicas de investigación cualitativa y cuantitativa que les permita enriquecer el diagnóstico del proyecto. Luego, en la tercera etapa, se realizan las llamadas devoluciones que son los talleres participativos donde se socializa el diagnóstico, pudiendo aplicarse técnicas participativas (pentalemas, flujogramas, árbol de problemas) de acuerdo a los objetivos del desarrollo de los talleres; y que conlleven a la discusión de propuestas colectivas que propongan soluciones a los problemas planteados durante esta etapa. En la siguiente etapa, se construirá de manera colectiva un escenario y rumbo a donde los actores sociales involucrados deseen ir; es decir, propuestas (política, económica, social y técnicamente) viables. Por último, las etapas ejecución y monitoreo, son permanentes y constantes; mientras que la de evaluación, será en momentos establecidos (antes de iniciar o durante el mismo y al finalizar) de acuerdo a la planificación del proyecto.

Así mismo, lo que se evalúa en un proceso participativo es el nivel de participación de los actores involucrados; es decir, el sentido de su participación. De acuerdo a la escalera de participación se practica desde dos enfoques: el enfoque de planificación (de arriba hacia abajo) y el de participación (de abajo hacia arriba). Ambos enfoques tienen seis niveles o escalones. En el enfoque de planificación, centrado en los funcionarios y técnicos, va de una participación normativa, pasando por una (2) consultiva técnica a una (3) estratégica, luego (4) una situacional, llegando a (5) una participativa, para alcanzar a (6) una democrática. En cambio, el enfoque de participación, que está más bien centrado en las personas (beneficiarios), va desde la (1) participación puntual donde solamente se recibe información del proyecto, (2) luego asistiendo a sesiones informativas en donde otro actor (Estado u otro) es quien tiene capacidad de control, (3) para después -en otro nivel- participan de manera consultiva, (4) seguida de una participación 
interactiva pero indirecta, (5) para pasar a una participación directa de co-gestión, conformando redes que comparten la toma de decisiones, y finalmente, (6) participando de las tomas de decisiones sobre todo el proceso en auto y co-gestión (Red-CIMAS, 2020). Con esta herramienta se podrá medir la participación y el tipo de involucramiento de los actores en el proceso. (Figura 1)

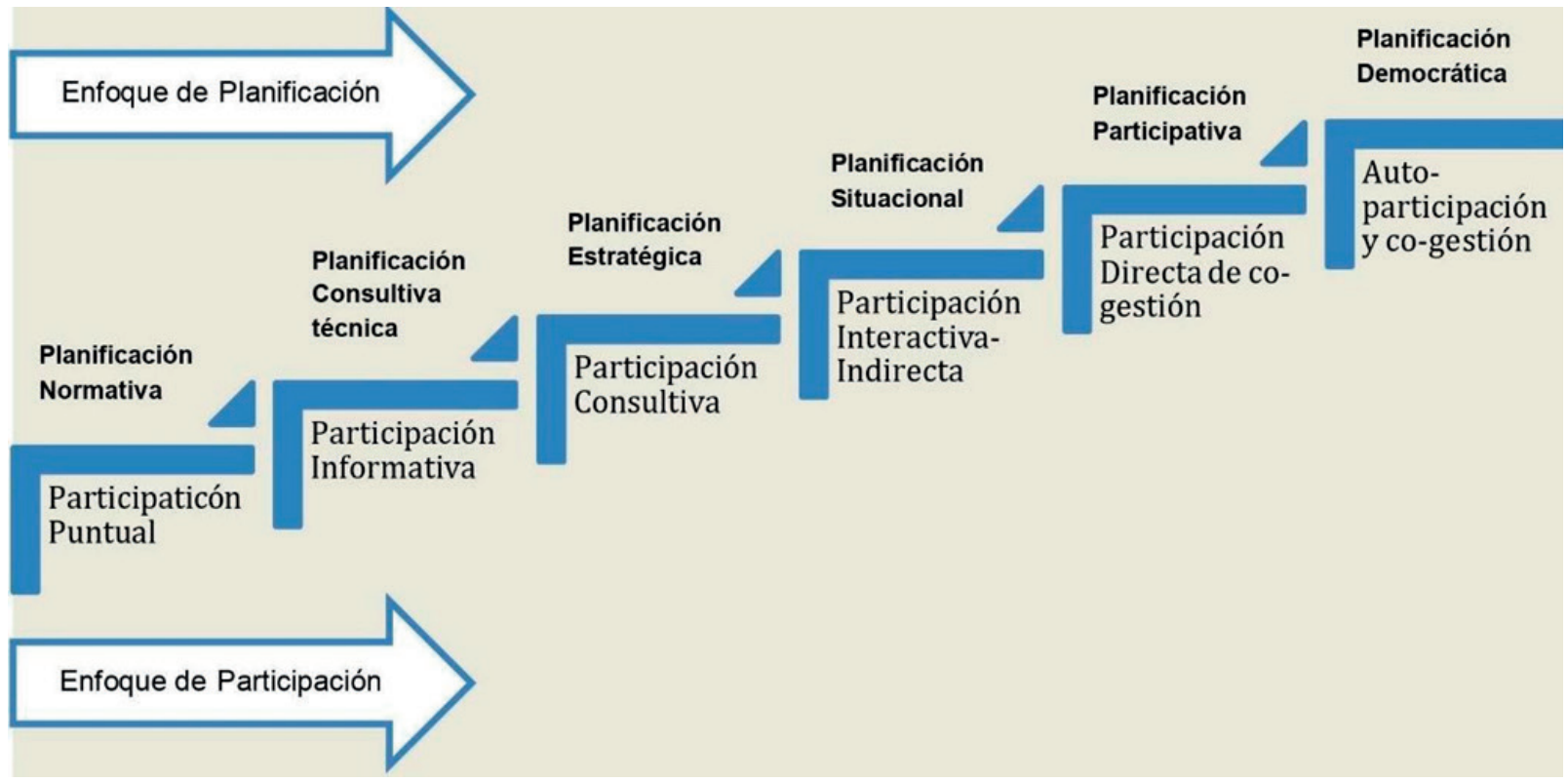

Figura 1. Escalera de la planificación y la participación

Fuente: Red CIMAS, 2020, p. 115.

\section{El enfoque Intercultural}

De acuerdo con Degregori \& Sandoval (2007), el enfoque intercultural tiene un origen en el concepto multiculturalismo, el cual busca una reivindicación del derecho a la diferencia e invita a la tolerancia, teniendo como experiencia lo sucedido en países multidiversos como EE.UU. donde se generaron comunidades homogéneas y cerradas entre sí. Mientras que, en América Latina y Canadá durante la década de los setenta surge el concepto de interculturalidad, inicialmente usado en el campo de la educación bilingüe, con la intención de eliminar el carácter desigual de los intercambios entre culturas distintas enfatizando en el proceso relacional entre ellas buscando el enriquecimiento mutuo de culturas conectadas por la globalización. En la década del noventa, desde la antropología como de los estudios subalternos se replantea las relaciones entre cultura y poder, cuyas aproximaciones metodológicas y estrategias etnográficas dieron paso del paradigma indigenista (profundamente marcado en las primeras décadas de la antropología peruana) a un paradigma intercultural. En este escenario se habla de una nueva relación de cultura y poder enfatizando las relaciones entre actores (sujeto-objeto, objeto-objeto) e intercambio entre culturas que nos invitan a la construcción de un nosotros diverso.

Por su parte, Tubino (2015) reflexiona acerca de la interculturalidad como una posibilidad de convivencia basada en el reconocimiento de la diversidad, como un primer paso para la universalización de los derechos humanos y la construcción de ciudadanía. Evitando así también conflictos interculturales en muchos casos ocasionados por la incomprensión del otro, donde ser ciudadano en un mundo intercultural debería ser teniendo derecho a mantener sus diferencias culturales: practicando su lengua originaria en el espacio público, formas propias de practicar la justicia y derecho a expresarse en espacios masivos de comunicación. (Ansión, 2007) 
Este enfoque aplicado a las metodologías participativas nos invita a reconocer e intercambiar los conocimientos y la diversidad cultural entre grupos sociales, así como comprender las lógicas de apropiación del territorio en el ámbito local plasmada en el conocimiento tradicional y lógicas locales dentro de un espacio determinado, del cual los demás actores sociales involucrados nos beneficiaremos del intercambio. Para ir más allá de la dicotomía campo-ciudad, espacio global-local y la relación sujeto-objeto en el diseño del proyecto y en la ejecución del proceso participativo.

\section{EI concepto glocal}

Lo glocal es un concepto desarrollado por la economía y posteriormente adoptado por las ciencias sociales. En la década de los ochentas fue usada en el marketing japonés, para hablar del mercado global sin renunciar a lo autóctono; es decir, lo local. Siendo luego difundido por Ulrich Beck y Roland Robertson (Garoz, 2016). Para Robertson (2000), el concepto glocalización puede reemplazar el concepto globalización, incluso brindar mayor precisión sobre las características de este último; donde «la noción japonesa particular aparece como una versión particular de un mismo fenómeno general» (p. 21). Esta categoría recoge el significado de la globalización resignificándolo en base al territorio y de lo local (Ferrero, 2006). Es un neologismo que busca permanencia a ambos conceptos, global y local, para hablar de una nueva geografía económica donde confluyen actores globales en escenarios locales puestos en la discusión del desarrollo rural (Moncayo, 2002). Como enfoque rompe con la separación binaria, por el contrario, plantea la inclusión de sectores antes excluidos en escenarios más bien hegemónicos para llevarlo a la práctica de proyectos (Reynoso, 2007). De esta manera también pone el dedo sobre la llaga y permite replantear modelos y corregir errores en dinámicas interculturales. (Bello, 2012)

Por último, el término es utilizado para identificar un mundo que ha dejado de ser un conjunto de sociedades independientes para consolidarse en un sistema articulado de subsistemas con mecanismos complejos y condicionados recíprocamente; y que, además, significa un reto para los actores sociales cuyos territorios integran distintas identidades y formas de vida, locales y globales, en el marco del desarrollo sostenible. (Murga-Menoyo \& Novo, 2017)

\section{Desarrollo etnográfico de la participación de actores en el TIDU}

En esta sección se describe el proceso participativo desarrollado en el TIDU 2019 ${ }^{4}$, tomando en cuenta los actores involucrados, la aplicación de las metodologías participativas, las acciones realizadas y el nivel de participación obtenido en el proceso.

Este proceso fue planificado y liderado por el equipo interdisciplinario de Urban-Andes ${ }^{5}$, encargado de movilizar a los actores locales que se involucraron en distintos momentos claves durante la realización TIDU que tuvo una duración de 10 días.

\section{Zonas de trabajo}

El área metropolitana de Ayacucho está conformada por cinco distritos: Ayacucho, Carmen Alto, San Juan Bautista, Jesús Nazareno y Andrés Avelino Cáceres Dorregaray. Para el desarrollo del TIDU se seleccionaron tres zonas de estudio: El Cerro Picota, La meseta de Mollepata y el

\footnotetext{
4 Cabe resaltar que la primera edición del TIDU fue en el 2018. También desarrollada en la ciudad de Ayacucho y con una duración similar de 10 días. Estos talleres se desarrollar en el marco del proyecto Urban-Andes a cargo del CCA, la Universidad de KU Leuven y la Universidad San Cristóbal de Huamanga.

5 El equipo local a cargo de la organización del TIDU estuvo conformado por Mónica Rivera, arquitecta, Vieyra Chipana, ingeniera civil, Maria Ñope, antropóloga y Luzia Ventura, bióloga. Durante el TIDU, tres tutores supervisaron el trabajo de las y los 18 participantes que elaboraron las propuestas de diseño urbano.
} 
Valle del río La Alameda. Estas zonas tienen como características similares ser lugares de baja consolidación urbana y deficiente planificación. (Tabla 1, figura 2)

Tabla 1. Resumen de los casos de estudio priorizados en el TIDU

\begin{tabular}{|ll|}
\hline Sector & Descripción \\
\hline El Cerro Picota & $\begin{array}{l}\text { Su ocupación empezó en } 1960 \text { y se intensificó luego de 1995. Son familias rurales migrantes, } \\
\text { hablan quechua y conservan sus costumbres. Realizan fiestas patronales, cultivan pequeños } \\
\text { huertos y tienen empleos precarios. Por su ubicación, son afectados por los deslizamientos en } \\
\text { épocas de lluvias que generan daños a las viviendas y vías. Algunos subsectores no cuentan con } \\
\text { saneamiento físico-legal ni acceso a servicios básicos de saneamiento. El gobierno local incentiva } \\
\text { la reforestación del cerro como una zona de protección ambiental para la ciudad. }\end{array}$ \\
\hline La meseta de Mollepata & $\begin{array}{l}\text { En las últimas dos décadas, la invasión informal de tierras y la especulación inmobiliaria en la } \\
\text { ciudad de Ayacucho incentivó su ocupación. La zona se divide en dos sectores: Mollepata Iy }\end{array}$ \\
& $\begin{array}{l}\text { Mollepata II. El TIDU trabajó con la Asociación Pro vivienda Ciudad de la Pacificación Mollepata II } \\
\text { (ADCIDEPA). La zona tiene un crecimiento horizontal y de baja densidad. Los principales problemas } \\
\text { son: la erosión de suelo, la deficiente gestión de residuos sólidos, el limitado acceso al agua } \\
\text { potable y eliminación de excretas, y la deficiente recolección y tratamiento de aguas residuales. }\end{array}$ \\
\hline El Valle del río La Alameda & $\begin{array}{l}\text { El río cruza y divide en dos la ciudad de Ayacucho. El río esta contaminado debido a la descarga } \\
\text { directa de aguas residuales domésticas sin tratamiento previo y la abundante concentración } \\
\text { de basura. Antes, el río era un lugar de encuentro para la vida social ayacuchana, pero ahora la } \\
\text { interacción entre la ciudad y el río es muy heterogénea; las fajas marginales han sido ocupadas por } \\
\text { viviendas reduciendo cada vez más las áreas de cultivos agrícolas. En la parte baja se encuentra la } \\
\text { Planta de Tratamiento de Aguas Residuales de la ciudad de Ayacucho. }\end{array}$ \\
\hline
\end{tabular}

Fuente: Elaboración propia

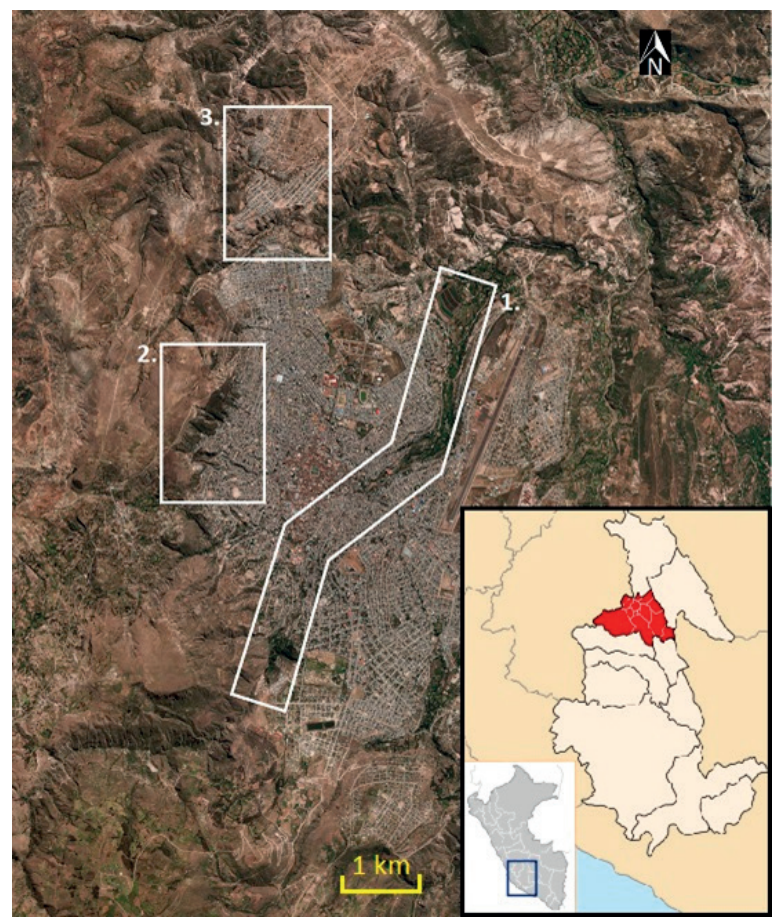

Figura 2. Ubicación de las zonas de intervención del TIDU: La Alameda (1); Sector Picota (2) y Mollepata (3)

Fuente: Adaptado del programa Urban-Andes, elaboración propia

\section{Sobre los actores involucrados}

Los actores involucrados en el TIDU fueron principalmente instituciones gubernamentales (gobierno regional, municipalidades provinciales y distritales) y no gubernamentales (ONGs, organizaciones sociales, colegios profesionales). La relación con la Municipalidad Provincial de Huamanga (MPH) fue de gran importancia para brindar mayor legitimidad y visibilidad al TIDU 
ante los diferentes actores locales. En coordinación con las gerencias municipales se logró el acercamiento con las organizaciones sociales de Picota, Mollepata y La Alameda.

A continuación, se listan los principales actores involucrados en el TIDU (ver figura 3). Para asegurar una amplia participación, se buscó la presencia de 1) organizaciones que trabajen sobre gestión territorial y saneamiento urbano, como COFOPRI y los colegios profesionales; 2) organizaciones que trabajen sobre la gestión del agua, como las direcciones regionales y la Empresa Prestadora de Servicios de agua SEDA Ayacucho; y 3) organizaciones sociales de las zonas de estudio 6 .

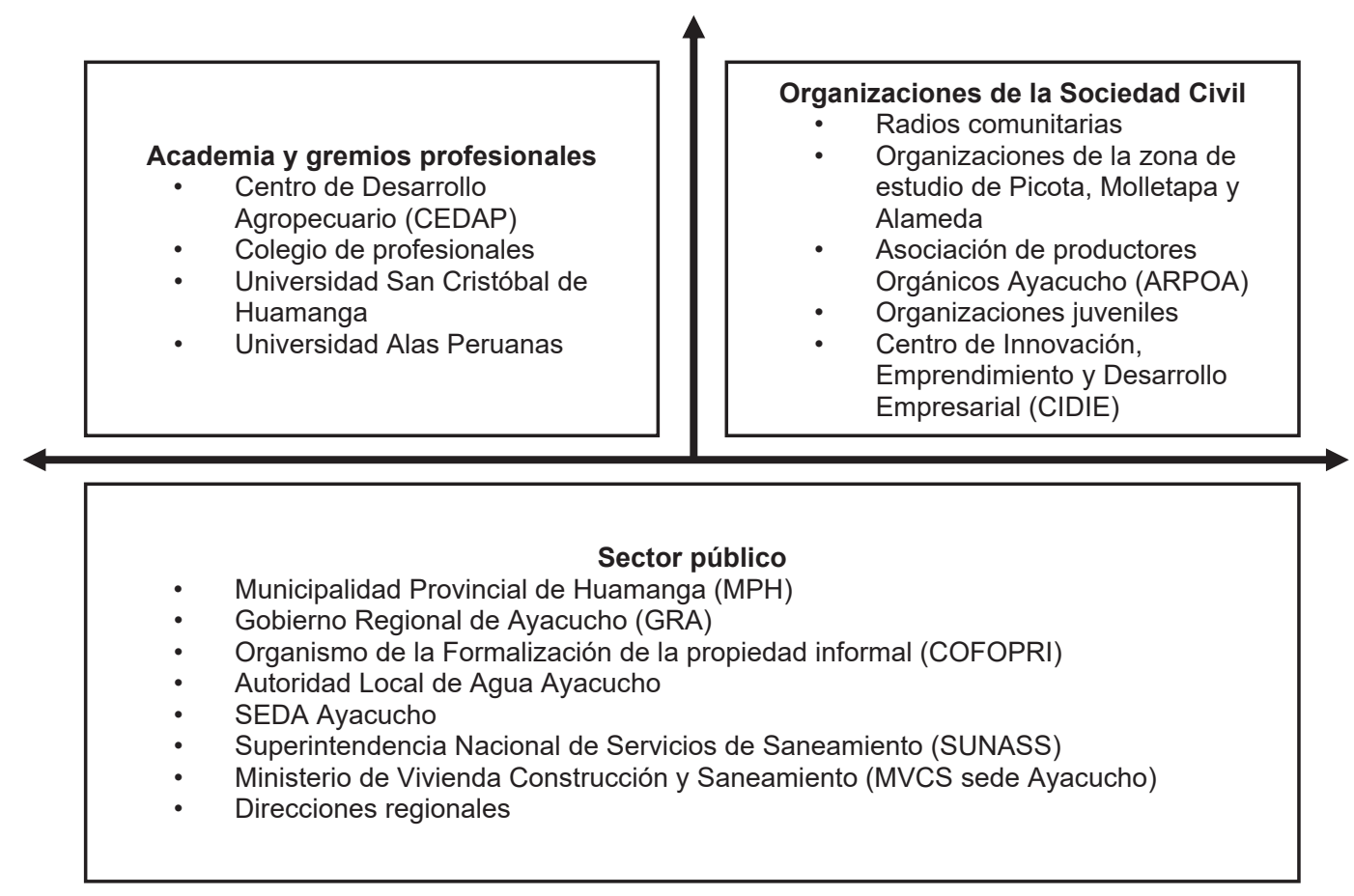

Figura 3. Relación de los principales actores involucrados en el TIDU

Fuente: Elaboración propia

La participación de las organizaciones sociales de las tres zonas de estudio fue clave para coordinar las salidas de campo previstas durante el TIDU. Asimismo, para facilitar la comunicación entre los pobladores y los participantes del TIDU, algunos de los facilitadores hablaban quechua, español e inglés.

\section{Metodología empleada}

Como parte de la preparación y ejecución del TIDU se emplearon métodos de análisis cualitativo, como el mapeo de actores, las entrevistas y diálogos y la observación de campo. Estos métodos permitieron levantar elementos de diagnóstico que alimentaron las propuestas de diseño urbano, para que respondan a problemáticas territoriales concretas e integren conocimientos locales.

Para asegurar un involucramiento dinámico de los actores se programaron, durante el TIDU, dos días para que puedan revisar y comentar los avances de los equipos a cargo de los diseños.

6 En Picota se trabajó principalmente con el Comité de Desarrollo Zonal, la radio comunitaria de Picota y con representantes de los barrios La Picota, Pueblo Libre y San Juan de la Picota. En Mollepata, se trabajó con la Asociación Pro- Vivienda Ciudad de la Pacificación Mollepata II (ADCIDEPA). En el Valle del río La Alameda se integraron instituciones involucradas en la gestión del agua. 
Al terminar el TIDU, Urban-Andes organizó talleres para presentar los diseños desarrollados por los participantes en las zonas de estudio.

\section{Nivel de participación de actores}

Viendo la elaboración de los diseños como producto final del TIDU, se puede destacar diversos niveles de participación alcanzados. En el peldaño superior, llamado gestión, resalta la participación de la KU Leuven, cuyos integrantes estuvieron a cargo de las propuestas. En el peldaño de la participación directa, o más colaborativa, resalta la participación de la UNSCH, algunas áreas de la MPH, CEDAP, SUNASS y algunas organizaciones comunales de las zonas de estudios. Estas instituciones influenciaron la orientación y el diseño de las propuestas a través de su interpretación del territorio y aportaron insumos que contribuyeron a la definición de las problemáticas. Así mismo, mantuvieron una comunicación constante con los participantes del TIDU durante su trabajo.

El peldaño siguiente, llamado participación interactiva, abarca a la mayoría de instituciones y organizaciones de la sociedad civil involucradas. Estás participaron en algunos momentos claves, pero no mantuvieron una comunicación tan seguida con el equipo supervisor y los participantes, como los actores mencionados anteriormente.

El peldaño siguiente, llamado participación consultiva, abarca a instituciones como los colegios profesionales, quienes ofrecieron aportes puntuales en el desarrollo de las propuestas de diseño. Finalmente, el peldaño llamado participación informativa abarca a instituciones que fueron informadas a través de reuniones, talleres o eventos, pero que no trataron de influir en las propuestas. (Figura 4)

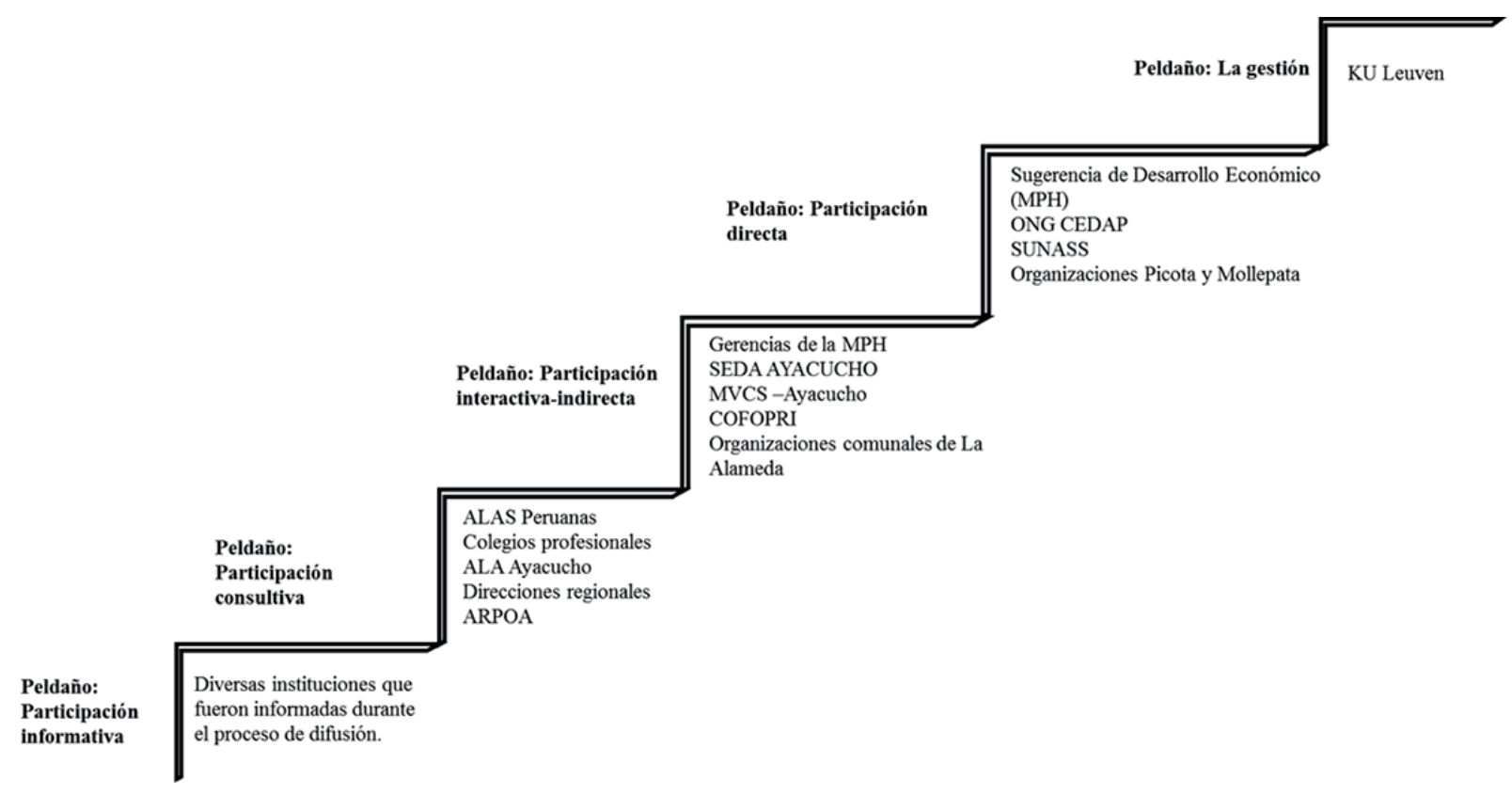

Figura 4. Nivel de participación de los actores involucrados

Fuente: Elaboración propia

\section{Diseños producidos en el TIDU}

Durante los 10 días que duró el taller se produjeron tres propuestas de diseños urbanos, una para cada zona de estudio. Estos diseños se desarrollaron en base a un concepto de intervención 
definido por cada equipo, incluyendo esquemas detallados para su implementación. Entre las características comunes a las propuestas se resalta: 1) concebir una ciudad más armónica con la naturaleza, conectando la población con las áreas verdes, ríos o cerros; 2) integrar intervenciones para mejorar la gestión del agua, por ejemplo, humedales o reforestación para mejorar la seguridad hídrica $^{7}$ y reducir los riesgos climáticos, sobre todo en las ocupaciones de laderas y quebradas.

Tabla 2.

Propuestas priorizadas en las zonas de intervención del TIDU

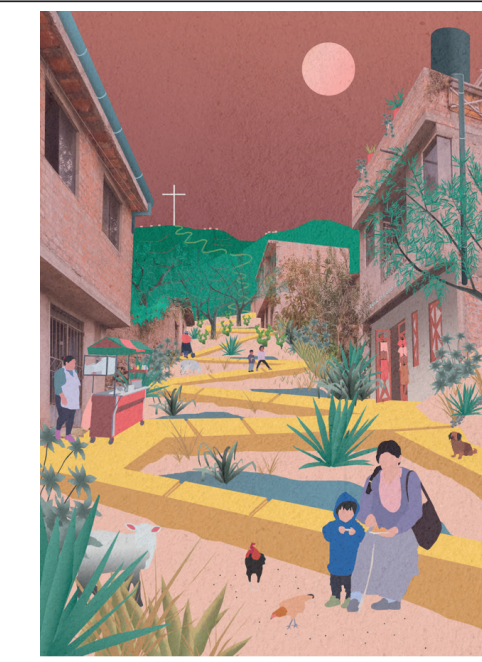

(1) En el Cerro Picota se propone realizar intervenciones de infraestructura verde para convertir la ladera en un protector de la ciudad, aumentando la retención del agua y mitigando el riesgo de deslizamiento.

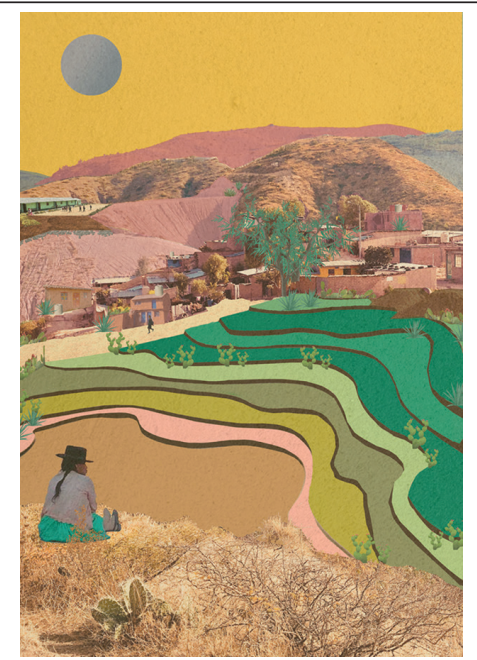

(2) En Mollepata se propone implementar humedales integrados al paisaje urbano que captan y tratan el agua residual, y en paralelo incrementan la cobertura de áreas verdes.

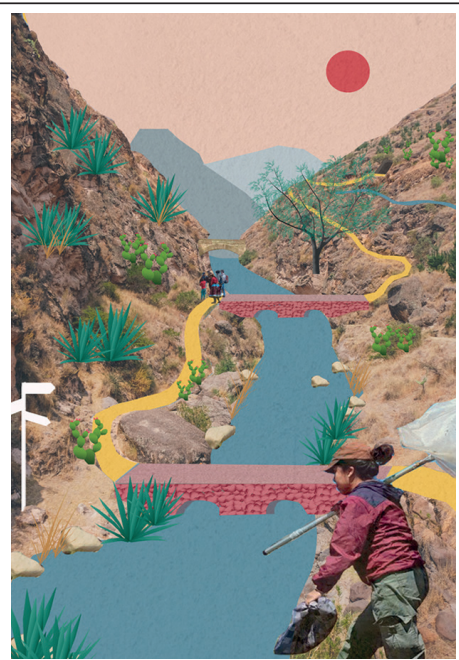

(3) En el Valle del río La Alameda se busca reverdecer y recuperar las fajas marginales del río para convertirla en zonas de encuentro e intercambio cultural. Esto implica integrar el río al paisaje urbano y disminuir la contaminación de ríos.

Fuente: Dibujos elaborados por los/as participantes del TIDU

\section{Discusión sobre la participación social en el TIDU}

A continuación, se discutirá el proceso participativo y su aplicación en contextos interculturales y glocales, como el de la ciudad de Ayacucho. Para ello, primero, se analiza la aplicación de las metodologías participativas en el ámbito local; segundo, se plantean los retos de los procesos participativos en un contexto intercultural y glocal. Por último se analizan los aportes de los procesos participativos al desarrollo local, enfatizando en su aplicación práctica.

\section{La aplicación de metodologías participativas en el ámbito local}

Las metodologías participativas, resultado de la conformación del paradigma crítico de las ciencias sociales en el siglo XX, nos invitan a la transformación de la sociedad. Si bien su aplicación comprende un conjunto de herramientas y técnicas, estas deben buscar dicha transformación mediante el involucramiento de los actores sociales en el ámbito local.

La aplicación de las metodologías participativas incluye técnicas como el mapeo de actores y talleres, junto con técnicas cualitativas tales como las entrevistas y la observación. Estas técnicas fueron empleadas durante el TIDU. No obstante, la aplicación de las metodologías

\footnotetext{
7 La seguridad hídrica es definida como «la provisión confiable de agua cuantitativa y cualitativamente aceptable para la salud, la producción de bienes y servicios y los medios de subsistencia, junto con un nivel aceptable de riesgos relacionados con el agua» (como se citó en Sadoff \& Muller, 2010, p. 14). En el caso de Ayacucho, las fuentes de agua para consumo humano son trasvasadas por el sistema del Proyecto Especial Río Cachi, un gran proyecto de infraestructura hidráulica para el desarrollo económico de la región, y del sistema de la cuenca del río Chiara.
} 
conlleva constantes desafíos que el equipo técnico de Urban-Andes debió sortear. En ese sentido, las metodologías participativas brindan pautas, pero su aplicación requiere creatividad para garantizar la participación de los actores sociales, y mejorar la aceptación social de las intervenciones urbanas en el mediano y largo plazo.

Además, la aplicación de las metodologías participativas tiene como objetivo final lograr incidencia en las políticas públicas, para lo cual la participación de los actores sociales tiene una relevancia gravitante. La participación contribuye a la disminución de las brechas sociales y asegura, en parte, la sostenibilidad social de un proyecto mediante la apropiación por los actores involucrados. Sin embargo, para que una propuesta tenga incidencia política como generar cambios en una política pública, existen grandes desafíos y retos. En el caso del TIDU diversos factores limitaron el impacto de las propuestas de diseños en las políticas urbanas a nivel local. Sin embargo, lo desarrollado en el presente artículo también da luces de que experiencias como estas puedan tener mayores resultados en el mediano o largo plazo.

Por último, su aplicación en el ámbito local debe centrarse en el capital social de sus participantes: valores compartidos, cultura, tradiciones, redes y expectativas de reciprocidad. Se debe concebir a los actores sociales como agentes transformadores de la sociedad. En ese aspecto el TIDU es también una oportunidad para generar conocimientos académicos que toman en cuenta las prácticas locales para aportar a la seguridad hídrica de la ciudad.

\section{Reflexión sobre los actores y sus intereses}

En el TIDU, los actores con mayor capacidad para sostener el proceso podrían ser la Municipalidad y la universidad. Sin embargo, la débil institucionalidad, el poco liderazgo y la baja legitimidad de las instituciones acrecientan la desconfianza por parte de los actores sociales. Debido a ello, el CCA no contó con aliados seguros para implementar las propuestas de diseño en el corto plazo. No obstante, dado el interés de ciertos actores (instituciones y organizaciones) se retomaron elementos surgidos en el TIDU para desarrollar otras propuestas específicas en la ciudad de Ayacucho o en la cuenca del río Cachi.

La participación tiene que considerar el capital social ${ }^{8}$ de las organizaciones. Esto da a conocer cuáles actores son capaces de sostener estos procesos. Esto hace de la participación un proceso complejo, pero necesario. Por ejemplo, la sociedad civil como agrupación de organizaciones o actores es heterogénea. A su vez, estas organizaciones pueden presentar contradicciones con los modelos democráticos que promueven, ocultando así prácticas verticales y controladoras que favorecen al bienestar individual antes que al colectivo. (Portocarrero 2006)

Los intereses del TIDU también se pueden ver sesgados por sus propios promotores. Por ejemplo, durante el taller los promotores se convirtieron en un actor más y no sólo en un facilitador del proceso, pues sus perspectivas son altamente influyentes en la orientación de las propuestas

Por último, la lección que se obtiene es que el tiempo para llevar a cabo un proceso participativo satisfactorio no necesariamente se enmarca en los tiempos que disponen las instituciones académicas y de la sociedad civil para realizar iniciativas como el TIDU.

\section{Retos de los procesos participativos en un contexto intercultural y glocal}

Los retos enfrentados para el involucramiento de actores durante el TIDU, implicaron, primero, reconocer la necesidad de un diálogo intercultural. Esta parte del supuesto de la horizontalidad, es decir, diferentes culturas interactúan de manera armónica, eliminando prejuicios de superioridad. 8 Aquí se enfatiza sobre todo el capital social organizacional o comunitario que implica normas, valores y procedimientos institucionales que aseguran la cooperación y la confianza entre personas y entre organizaciones. 
Lo intercultural en el Perú supone evidenciar que las diferencias étnicas muchas veces están relacionadas con las condiciones de desigualdad y exclusión a los cuales han estado orillados las poblaciones rurales. Por ello, lo intercultural no sólo es pertinente para formular proyectos de desarrollo y procesos participativos, sino también políticas locales (Thorp \& Paredes, 2010). La diversidad cultural es un aspecto que todavía define a la ciudad de Ayacucho, en donde, la evolución de las relaciones de poder fueron excluyendo sistemáticamente a la población migrante pobre. Esto se refleja en la configuración de la ciudad (su ocupación física); las poblaciones más pobres ocupan las periferias.

En el caso del TIDU, encontramos que hacer comunicación en un contexto intercultural es más complejo pero factible. Sin embargo, al involucrar a diversos actores toma mucho más tiempo. Ejemplo de ello es que los espacios aperturados y las técnicas empleadas no fueron suficiente para lograr una integración entre los actores. Esto se explica en parte por las tensiones existentes entre los actores. Por ejemplo, las organizaciones sociales tienen un reclamo sobre el saneamiento físico y legal, el cual no puede ser atendido en el marco del TIDU por las autoridades públicas. Por otro lado, persiste un desafío al tratar de vincular el conocimiento académico con los plazos con que se elaboran políticas públicas.

El siguiente desafío está relacionado a lo local frente a lo glocal. Por local se entiende tanto el espacio como el proceso de territorialización de los actores en su entorno, es decir, la construcción de comunidades y colectividades en el territorio (Coraggio, 2003). Estas poblaciones guardan historias, experiencias y conocimientos que pueden llegar a ser determinantes en los proyectos de desarrollo, pero que no siempre se incorporan a fin de simplificar los procesos participativos.

Lo local cuestiona los modelos homogeneizadores de la cultura hegemónica y del desarrollo. En tanto, lo glocal sienta puntos de encuentro entre lo global como manifestación de procesos globalizados y lo local como valorador de elementos diferenciadores (una identidad propia).

Bajo esa orientación, se resalta la necesidad de proyectos de desarrollo que asuman el reto de pensar y responder localmente a los procesos globales de urbanización, la inseguridad hídrica y el cambio climático. Desde Urban-Andes, la estrategia para abordar simultáneamente estas problemáticas es la elaboración de propuestas de diseño urbano, en las cuales se plasman las visiones, los conocimientos y las expectativas de los actores locales. Experiencias similares surgen cada vez más, inclusive desde las organizaciones,

caracterizados por decisiones y acciones que incluyen a habitantes locales y formas más cuidadosas de relación con el entorno. Estos procesos de cogestión incluyen o aspiran a una amplia participación social, donde la diversidad de visiones, el diálogo de saberes y la acción colectiva permitan mantener o generar formas de vida más sustentables y justas. (Hensler et al., 2019, p. 236)

Esta relación entre lo local y lo global también hace que las herramientas y metodologías participativas tengan más de un propósito, pues consideran sus sinergias. En Ayacucho, esto puede ser entendido desde múltiples aristas, como el peso histórico de una ciudad virreinal (racista y elitista) o las secuelas del conflicto armado interno. Sin duda, estos factores confluyeron para crear una sociedad fragmentada y desigual, carente de instituciones capaces de generar confianza y sostener procesos participativos.

Otro desafío es el entendimiento de la participación por los propios actores. Si bien UrbanAndes buscó superar la dicotomía de una participación de arriba-abajo o de abajo-arriba, en la práctica esto fue complejo. Por un lado, trató de incidir en las autoridades municipales sobre 
el valor de la gestión colaborativa, pero no consiguió asegurar la voluntad política real ni la confianza plena de la población participante del proceso. Y, por otro lado, trató de consolidar una conciencia colectiva, pero los conflictos y las tensiones entre las organizaciones gubernamentales, la sociedad civil y el sector privado no lo garantizaron. Aun así, el hacer una intervención de doble vía permitió aprovechar los espacios de participación institucionalizados, y visibilizar el rol de los actores como sujetos colectivos capaces de transformar el orden social y político. (Canto, 2016)

La incorporación de actores en el quehacer público es clave, sobre todo en el diálogo cienciapolítica, que busca deconstruir la separación entre lo científico/técnico y lo político. De esa manera, aporta a la mejor gestión del territorio urbano y del agua. Esto se ve reflejado en el diseño y aplicación del TIDU, el cual se convierte en una herramienta de planificación para los gobiernos locales y las organizaciones sociales en Ayacucho. Desde ahí, se generan propuestas de diseño urbano integrales, con el rasgo distintivo de ser creaciones colaborativas multiactor y multinivel.

Por último, se plantea el desafío de los gobiernos subnacionales y la participación social en los procesos locales. En este nivel es donde se debe promover la participación, así como las condiciones que permitan que la población participe no sólo en cantidad sino en calidad. Bajo esa perspectiva, la participación no se restringe a los parámetros ideados por las instituciones convocantes, sino tiene flexibilidad para integrar condiciones que posibilitan una participación plural donde los resultados no estén predeterminados.

Cuando se observan las formas de participación impulsadas por las municipalidades, como el presupuesto participativo o la consulta ciudadana, se nota que son espacios debilitados. Por ejemplo, en la ciudad de Ayacucho, tanto las organizaciones de Picota y Mollepata se retiraron del presupuesto participativo alegando que no estaban siendo escuchadas sus necesidades y propuestas. Como equipo, Urban-Andes tuvo la oportunidad de participar en dos talleres del presupuesto participativo en Picota. La sorpresa fue que parecían una cancha sin árbitro; por un lado, las organizaciones enumerando su lista de proyectos, unos más exhaustivos que otras, y de otro lado, la municipalidad con sus propias exigencias para la población. Estas situaciones reflejan lo complejo que es sentar un diálogo intercultural, horizontal y efectivo en el quehacer público9. Sin embargo, también permiten identificar aspectos de mejora para lograr un mayor liderazgo de los actores sociales.

\section{Aportes de las metodologías participativas al desarrollo local}

El estudio presentado aquí brinda aportes al fortalecimiento de la gobernanza. La participación como base de la gobernanza abre la oportunidad para empoderar a individuos y colectividades, promoviendo la defensa de derechos, los cuales muchas veces son cuestionados o vulnerados por la falta de diálogo, la imposición de prácticas antidemocráticas o por el modelo económico. A causa de estas problemáticas surgen múltiples conflictos sociales y ambientales a nivel nacional.

Por ello, es de suma importancia impulsar procesos desde la localidad, reconociendo las diferencias culturales, históricas, estructurales así como su interseccionalidad. En esa línea, el involucramiento de actores en el TIDU trató de dar voz a la población y cuestionar el rol pasivo de los gobiernos locales, quienes, en ocasiones no lograron superar formas antañas de relacionamiento con la población basadas en prácticas de clientelaje o de manipulación política.

9 Tomando como referencia a Héctor Bejar (2011) esto también se puede extrapolar a la construcción de políticas sociales participativas, pues hasta ahora el Estado no logra garantizar el derecho a la participación. El déficit de participación es más agudo aun cuando se analiza el caso de los pueblos originarios de la Amazonía 
Cabe resaltar que el proceso participativo aquí descrito no hubiese sido posible bajo las condiciones actuales que nos plantea la pandemia de la COVID-19 ${ }^{10}$. La pandemia una vez más ha expuesto las desigualdades y la pobreza originada de una inadecuada planificación urbana, así como de la marginación y exclusión sistemática de los sectores poblacionales más vulnerables. Por su lado, los gobiernos y diversas instituciones buscan continuar con los procesos participativos o de consulta, cada vez más apoyados en herramientas digitales. Lamentablemente, esta no parece ser una solución dada la brecha de conectividad persistente en las ciudades, y sobre todo en el ámbito rural. A esto se suman las desigualdades de género y étnicas, que sin duda impactan de manera diferenciada en los distintos sectores de la sociedad y en el quehacer cotidiano de las personas.

Por ello, aquí se reafirma la importancia de aplicar metodologías participativas en los proyectos de desarrollo urbano. Cada vez más hay ejemplos de trabajos colaborativos que deberían ser aprovechados por las instituciones gubernamentales. Estas son alternativas duraderas. Además, están sustentadas en la evidencia y equilibradas de colaboración, mejorando así las relaciones de confianza y legitimidad de los actores a nivel local. (Hensler et al., 2019)

\section{Conclusiones}

Los procesos participativos locales en la producción de la ciudad, a partir de la experiencia del TIDU, demuestran que la voluntad y la aplicación de metodologías participativas no son suficientes en sí mismas para alcanzar una mayor participación. Puesto que, requiere de compromisos concretos y del sentido crítico de los diferentes actores capaces de realzar la voluntad colectiva en aras de mejorar la calidad de vida. El análisis de la experiencia evidencia la importancia de la variable intercultural en el diálogo y en la interacción de actores durante todo el transcurso del TIDU. Debido a que la ciudad de Ayacucho conserva una vasta riqueza cultural y un fuerte arraigo a prácticas andinas, lo intercultural va más allá del encuentro entre culturas sino que se convierte en una apuesta política. El proceso participativo del TIDU, en línea con la IAP, tuvo como reto deconstruir las formas de participación tradicionales, en donde los actores son sujetos pasivos que reciben información, para incentivar la reflexión-acción entre instituciones y actores de la sociedad civil. Sin embargo, hubo dificultades para propiciar una integración plena de los actores involucrados.

De igual manera, en la investigación se destaca el concepto de glocalización, porque este resalta el nexo entre el actuar local y los procesos globales como el cambio climático, la migración humana o la pérdida de biodiversidad. En Ayacucho, por ejemplo, los problemas del agua tendrían que inspirar y valorar soluciones arraigadas a la cosmovisión andina, como la siembra y cosecha del agua.

A partir de esta experiencia, se reconoce que la participación social en la gestión y planificación territorial todavía es insuficiente, sobre todo en el espacio local y regional en el que se desarrolló el TIDU. La debilidad de las instituciones gubernamentales y no gubernamentales dificulta las relaciones, especialmente, con las organizaciones sociales de los sectores más vulnerables. Frente a ello, Urban-Andes, por un lado, trabajó con los gobiernos locales y, por otro lado, con la sociedad civil, especialmente con las organizaciones sociales a nivel microlocal. Sin embargo, los resultados logrados no son fáciles de medir en el corto plazo.

La participación es un proceso iterativo que implica dialogar, negociar y consensuar en torno a los intereses individuales y colectivos. Por ello es clave la construcción de sujetos colectivos

10 El escenario nacional actual es complejo; tenemos un país duramente golpeado por la pandemia y un cambio de gobierno por delante. 
fortalecidos que representen nuevos liderazgos, capaces de sostener los procesos participativos, así como de identificar acciones a favor del bienestar social.

Finalmente, esperamos con esta investigación aportar a la reflexión académica sobre las metodologías participativas que, en muchas ocasiones, quedan como un mero instrumento a aplicar, mas no como una oportunidad para propiciar mejores condiciones de vida.

\section{Referencias}

Alvarado, Lusmidia y García, Margarita. (2008). Características más relevantes del paradigma sociocrítico: su aplicación en investigaciones de educación ambiental y de enseñanza de las ciencias realizadas en el Doctorado de Educación del Instituto Pedagógico de Caracas. Sapiens. Revista Universitaria de Investigación, 9 (2), 187202.

Ansión, Juan. (2007). La interculturalidad y los desafios de una nueva forma de ciudadanía. En E. A. Ansión, Educar en ciudadanía intercultural (pp. 37-62). Lima: Pontificia Universidad Católica del Perú.

Béjar, Héctor. (2011). Justicia social, política social. Lima: ACHEBE Ediciones.

Bello, Daniel. (2012). Alianza Estratégica Aymaras sin Fronteras: Una respuesta territorial a los desafíos de la localización. T'inkazos, (32), 147-164.

Canto Chac, M. (2008). Gobernanza y participación ciudadana en las políticas públicas frente al reto del desarrollo. Politica y cultura, (30), 9-37.

Canto, Manuel. (2016). Las políticas públicas participativas, las organizaciones de base y la construcción de espacios públicos de concertación local. https://bit.ly/2ZXrak8

CEPAL (1998). Panorama social de América Latina. Santiago de Chile: Naciones Unidas.

CLAD (2009). Carta Iberoamericana de Participación Ciudadana en la Gestión Pública. https://bit.ly/3BNmWs2

Coraggio, José. (2003). Las políticas públicas participativas: ¿obstáculos o requisito para el Desarrollo Local? https://bit.ly/3GQxyu 9

Degregori, Carlos Iván y Sandoval, Pablo. (2007). Dilemas y tendencias en la antropología peruana del paradigma indigenista al paradigma intercultural. En C. I. Degregori, \& P. Sandoval (Eds.). Saberes periféricos: ensayos sobre la antropología en América Latina (pp. 19-72). Lima: Instituto de Estudios Peruanos, Instituto Francés de Estudios Andinos.

Fals-Borda, Orlando. (1970). Ciencia propia y colonialismo intelectual. México D.F.: Editorial Nuestro Tempo.

Ferrero, Mariano. (2006). La glocalización en acción: Regionalismo y paradiplomacia en Argentina y todo el Cono Sur Latinoamericano. Revista Electrónica de Estudios Internacionales. https://bit.ly/3k5prjD

Freire, Paulo. (1970). Pedagogía del oprimido. México: Siglo Veintiuno Editores.

Garoz López, Guillermo. (2016). DEBATE 21. La glocalización como respuesta al pensamiento único. https://bit. ly/31AOULz

Habermas, Jürgen (1994). La teoría de la acción comunicativa, complementos y estudios previos. Madrid: Cátedra.

Hensler, Lone; Merçon, Juliana, Gonzáles-González, Rodrigo; Estrada, Ingrid; Paradowska, Krystyna; Bravo, Leticia y Cesareo, Valeria. (2019). Metodologías participativas para la cogestión del territorio. Una experiencia de aprendizaje colectivo en Veracruz, México. En Pablo Paño, Romina Rébola y Mariano Suárez (Eds.), Procesos y metodologías participativas. Reflexiones y experiencias para la transformación social (pp. 235260). Montevideo: CLACSO - UDELAR.

Instituto Nacional de Estadística e Informática. (2018). Perú: Perfil sociodemográfico (informe nacional). Lima: INEI.

Kliksberg, B. (1998). Seis tesis no convencionales sobre participación. Revista instituciones y desarrollo, (2), 131170 .

Lois, Ianina (2017). La investigación-Acción (I+A) y la investigación Acción Participativa (IAP): un recorrido posible entre el conocimiento y la praxis. https://bit.ly/3BXhYtb 
Merçon, Juliana (2018). Participatory action research and decolonial studies. Critical mirrors. Decolonial Education in the Americas: Lessons of Resistance, Pedagogies of. Latin American Philosophy of Education Journal, (3), 20-29.

Moncayo, Edgard (2002). Glocalización: Nuevos enfoques teóricos sobre el desarrollo regional (sub nacional) en el contexto de la integración económica y de la globalización. Desafíos, 7, 50-99.

Murga-Menoyo, M. A. y Novo, Maria. (2017). Sostenibilidad, desarrollo «glocal» y ciudadanía planetaria. Referentes de una pedagogía para el desarrollo sostenible. Teoría de la Educación Revista Interuniversitaria, 29(1), 55-78. https://bit.ly/3GNgkhn

Popkewitz, T. (1998). Paradigma e ideología en investigación educativa. Las funciones sociales del intelectual. Madrid: Mondadori.

Quijano, Aníbal. (1999). Colonialidad del poder, cultura y conocimiento en América Latina. Dispositio, 24 (51), $137-148$.

Red CIMAS. (2020). Estrategias Transformadoras. Cómo potenciar la creatividad colaborativa desde los movimientos y procesos sociales. Madrid: CIMAS.

Reynoso, Elaine. (2007). Entre lo global y local: hacia la construcción del contexto glocal. RED POP-UNESCO y IV Taller “Ciencias, Comunicación y Sociedad". Recuperado en 05 octubre de 2020, de https://bit.ly/3BFjU9m

Robertson, Roland. (2000). Glocalización: tiempo-espacio y homogeneidad heterogeneidad. Zona Abierta, (92-93), 221.

Sandoval, Carlos; Sanhueza, Andrea y Williner, Alicia. (2015). La planificación participativa para lograr un cambio estructural con dignidad: Las estrategias de participación ciudadana en los procesos de planificación escalar. Santiago de Chile: CEPAL.

Suarez, Mariano y Meneses, Fabiana. (2019). Metodologías participativas: Orígenes y características del proceso. En Alejandro Novoa (Coord.), Conocer lo Social III: Las metodologías emergentes (pp. 86-112). Salto: Fundación de Cultura Universitaria.

Tubino, Fidel (2015). La interculturalidad en cuestión. Lima: Pontificia Universidad Católica del Perú.

Thorp, Rosemary y Paredes, Maritza (2010). Las desigualdades étnicas y sus consecuencias para la política social: el caso del Perú. En Felipe Portocarrero, Enrique Vásquez y Gustavo Yamada (Eds.). Políticas sociales en el Perú: nuevos desafíos (pp. 21-49). Lima: Pontificia Universidad Católica del Perú, Universidad del Pacífico, Instituto de Estudios Peruanos y Red para el Desarrollo de las Ciencias Sociales en el Perú.

Sadoff, Claudia y Mike Muller (2010). La Gestión del Agua,la Seguridad Hídrica y la Adaptación al Cambio Climático: Efectos Anticipados y Respuestas Esenciales. https://bit.ly/3EP0ctV

Presentado: 04/07/2021

Aceptado: 30/09/2021

Publicado online: $27 / 12 / 2021$ 